\title{
Global fluctuation formulas and universal correlations for random matrices and log-gas systems at infinite density
}

\author{
P.J. Forrester* \\ Department of Mathematics \\ University of Melbourne \\ Parkville, Victoria 3052 \\ Australia
}

\begin{abstract}
It is shown how the universal correlation function of Brézin and Zee, and Beenakker, for random matrix ensembles of Wigner-Dyson type with density support on a finite interval can be derived using a linear response argument and macroscopic electrostatics. The analogous formula for ensembles of unitary random matrices is derived using the same method, and the corresponding global fluctuation formula for the variance of a linear statistic is presented. The result for the universal correlation is checked using an exact result for the finite system two-point correlation in the Dyson circular ensemble at all even $\beta$.

PACS numbers: $72.10 . \mathrm{Bg}, 05.40 .+\mathrm{j}, 05.60 .+\mathrm{w}$
\end{abstract}

\section{Introduction}

A large portion of random matrix theory is concerned with the eigenvalue probability density function

$$
\prod_{l=1}^{N} e^{-\beta V\left(x_{l}\right)} \prod_{1 \leq j<k \leq N}\left|x_{k}-x_{j}\right|^{\beta}, \quad x_{j} \in \mathbf{R}
$$

where $\beta=1,2$ or 4 according to the matrices being real symmetric, Hermitian or quaternion real respectively (see e.g. [1]). An alternative interpretation of (1.1) is as the Boltzmann factor

\footnotetext{
${ }^{*}$ Supported by the Australian Research Council
} 
of a one-component log-potential Coulomb system confined to a line with potential energy

$$
\sum_{l=1}^{N} V\left(x_{l}\right)-\sum_{1 \leq j<k \leq N} \log \left|x_{k}-x_{j}\right|
$$

The two body term in (1.2) is the electrostatic energy of the Coulomb repulsion between like charges, while the one body potential acts to confine the particles to some interval of the real line.

In particular, consider the situation in which $V(x)$ is infinite outside an interval $(a, b)$ so that

$$
x_{j} \in(a, b)
$$

This setting has recently been discussed by Brézin and Zee [2], in the special case $\beta=2$ and $V(x)$ an even polynomial about the centre of the interval (see also [3]), and by Beenakker [4], for general $\beta$ and $V(x)$. These authors have found that the two-point correlation, $\rho^{T}\left(x, x^{\prime}\right)$ say, satisfies the universal formula

$$
\begin{aligned}
\lim _{N \rightarrow \infty} \frac{N}{b-a} & \int_{x^{\prime}}^{x^{\prime}+(b-a) / N} d t \rho_{N}^{T}(x, t) \\
& =-\frac{1}{\beta \pi^{2}\left(x-x^{\prime}\right)^{2}} \frac{(a+b)\left(x+x^{\prime}\right) / 2-a b-x x^{\prime}}{\left[(x-a)(b-x)\left(x^{\prime}-a\right)\left(b-x^{\prime}\right)\right]^{1 / 2}}, \quad x \neq x^{\prime}
\end{aligned}
$$

Remark: The function $\rho^{T}\left(x, x^{\prime}\right)$ above equals $-T_{2}\left(x, x^{\prime}\right)$ in the notation of Beenakker [4].

The l.h.s. of (1.4) corresponds to the two point correlation in the infinite density limit, smoothed over the average interparticle spacing.

As a consequence of (1.4), Beenakker [4] has derived the global fluctuation formula

$$
\begin{aligned}
& \lim _{N \rightarrow \infty} \operatorname{var}\left[\sum_{j=1}^{N} f\left(x_{j}\right)\right] \\
& \quad=\frac{1}{\beta \pi^{2}} \mathcal{P} \int_{a}^{b} d x \int_{a}^{b} d x^{\prime}\left[\frac{\left(x^{\prime}-a\right)\left(b-x^{\prime}\right)}{(x-a)(b-x)}\right]^{1 / 2} \frac{f(x)}{x-x^{\prime}} \frac{d}{d x^{\prime}} f\left(x^{\prime}\right)
\end{aligned}
$$

where $\mathcal{P}$ denotes the principal part, for the variance of a linear statistic.

The study of random matrix ensembles with density support confined to a finite interval follows the study [5-9] of ensembles with density support on the half interval $[0, \infty)$, which are of relevance to fluctuation phenomena in mesoscopic systems. In this setting it is not necessary to consider an infinite density limit. Rather, a scale can be chosen so that the number of eigenvalues near the "edge" $x=0$ remains finite [7] in the $N \rightarrow \infty$ limit. The asymptotic expansion of the corresponding two-point correlation satisfies a formula analogous to (1.3):

$$
\rho^{T}\left(x, x^{\prime}\right) \sim-\frac{1}{2 \pi^{2} \beta \sqrt{x x^{\prime}}} \frac{x+x^{\prime}}{\left(x-x^{\prime}\right)^{2}}
$$


for the leading non-oscillatory behaviour. Beenakker [9] (see also [10]) has used (1.5) to derive the analogue of (1.4) for the fluctuation of a linear statistic.

Recently [11] a new derivation of (1.5) has been given, applicable to all log-potential Coulomb systems in their conductive phase, which is based on macroscopic electrostatics and linear response theory. It is our purpose herein to first rederive (1.4) using the method of [11], and then to consider the analogues of (1.4) and (1.5) for unitary random matrices (or equivalently log-gas systems confined to a circle). For the one-component log-gas on a circle at even $\beta$, the two point correlation has been evaluated exactly [12] in terms of a $\beta$-dimensional integral for all particle numbers $N$, which allows an explicit verification of the analogue of (1.4).

\section{Macroscopic electrostatic argument}

Our objective in this section is to derive (1.4). For simplicity of notation we consider the symmetric case $a=-b$ and scale the variables $x \mapsto b x$ and $x^{\prime} \mapsto b x^{\prime}$, so that the spectrum has support in $(-1,1)$. The r.h.s. of $(1.4)$ then reads

$$
-\frac{1}{\beta \pi^{2}\left(x-x^{\prime}\right)^{2}} \frac{1-x x^{\prime}}{\left[\left(1-x^{2}\right)\left(1-x^{\prime 2}\right)\right]^{\frac{1}{2}}}, \quad x \neq x^{\prime}
$$

There is no loss of generality is specializing to this case as the r.h.s. of (1.4) can be reclaimed from (2.1) by rescaling the variables $x \mapsto 2(x-(a+b) / 2) /(b-a)$ (and similarly $\left.x^{\prime}\right)$. Rather than restricting ourselves to systems with probability density function (1.1) we will consider any log-potential Coulomb system confined to the interval $(-1,1)$ which consists of point particles and is in a conductive phase.

Our starting point is the linear response argument of [11], which says that if the system is perturbed by adding an external charge $\delta q$ at a point $\overrightarrow{r^{\prime}}$, then

$$
<\Phi(\vec{r})>-<\Phi(\vec{r})>_{0}=-\beta \delta q<\Phi(\vec{r}) \Phi\left(\overrightarrow{r^{\prime}}\right)>^{T}
$$

where $\Phi(\vec{r})$ is the potential at the point $\vec{r}$ due to the induced charge distribution, $<>_{0}$ refers to the value of the average before the perturbation and $<A B>^{T}=<A B>_{0}-<A>_{0}<B>_{0}$.

To obtain from (2.2) the charge density- charge density correlation

$$
<\sigma(x) \sigma\left(x^{\prime}\right)>^{T}
$$

we hypothesize that in the high density $N \rightarrow \infty$ limit, the system behaves as a conducting 
interval from -1 to 1 along the $x$-axis, obeying the laws of two-dimensional macroscopic electrostatics. Now, for a point $x$ on the $x$-axis, macroscopic electrostatics gives

$$
E_{y}^{+}(x)-E_{y}^{-}(x)=2 \pi \sigma(x)
$$

where the $y$-components of the electric fields are given in terms of the potential by

$$
E_{y}^{+}(x):=\left.\frac{\partial}{\partial y} \Phi(\vec{r})\right|_{y=0^{+}} \quad E_{y}^{-}(x):=\left.\frac{\partial}{\partial y} \Phi(\vec{r})\right|_{y=0^{-}}
$$

Hence

$$
\begin{aligned}
-\beta \delta q & <\sigma(x) \sigma\left(x^{\prime}\right)>^{T} \\
= & -\beta \delta q \frac{2}{(2 \pi)^{2}}\left(<E_{y}^{+}(x) E_{y^{\prime}}^{+}\left(x^{\prime}\right)>^{T}-<E_{y}^{+}(x) E_{y^{\prime}}^{-}\left(x^{\prime}\right)>^{T}\right) \\
= & \frac{2}{(2 \pi)^{2}}\left(\left.\frac{\partial}{\partial y} \frac{\partial}{\partial y^{\prime}}\left(<\Phi(\vec{r})>-<\Phi(\vec{r})>_{0}\right)\right|_{y=0^{+}, y^{\prime}=0^{+}}\right. \\
& \left.-\left.\frac{\partial}{\partial y} \frac{\partial}{\partial y^{\prime}}\left(<\Phi(\vec{r})>-<\Phi(\vec{r})>_{0}\right)\right|_{y=0^{+}, y^{\prime}=0^{-}}\right)
\end{aligned}
$$

where to obtain the first line we have used the symmetry

$$
<E_{y}^{+}(x) E_{y^{\prime}}^{s}\left(x^{\prime}\right)>^{T}=<E_{y}^{-}(x) E_{y^{\prime}}^{-s}\left(x^{\prime}\right)>^{T}
$$

for $s=+,-$ and to obtain the second line (2.5) and (2.2) have been used.

By the hypothesis of the applicability of macroscopic electrostatics,

$$
<\Phi(\vec{r})>-<\Phi(\vec{r})>_{0}
$$

is simply the potential at a point $\vec{r}$ due to the charge induced on the conducting interval $(-1,1)$ by the external charge $\delta q$. The calculation of this potential is a standard problem in $2 d$ electrostatics [13, Chapter 4, exercise 5]. One first considers the potential at $\vec{r},|\vec{r}|>1$, due to a unit charge at $\overrightarrow{r^{\prime}},\left|\overrightarrow{r^{\prime}}\right|>1$, and a conducting unit disk centred at the origin. By the method of images, this potential is

$$
\Phi\left(z, z^{\prime}\right)=-\log \left(\left|z^{\prime}-z\right| /\left|z^{\prime}-1 / z^{*}\right|\right)-\log \left|z^{\prime}\right|
$$

where $z\left(z^{\prime}\right)$ is the complex coordinate corresponding to $\vec{r}=(x, y)\left(\overrightarrow{r^{\prime}}=\left(x^{\prime}, y^{\prime}\right)\right)$, and it is assumed the conductor carries no net charge. Then one maps the exterior of the unit disk to the plane, with a cut from -1 to 1 along the $x$-axis by the conformal mapping

$$
w=z+\frac{1}{z} \quad \text { and thus } \quad z=w+\left(w^{2}-1\right)^{1 / 2}
$$


where the principal branch of the square root is chosen.

Using (2.8) to substitute in (2.7) for $z$ (and similarly $z^{\prime}$ ) we obtain the potential at $\vec{r}$ due to the charge induced on the conducting interval and due to the external charge at $\overrightarrow{r^{\prime}}$. Subtracting the potential due to the external charge we therefore have

$$
\begin{gathered}
<\Phi(\vec{r})>-<\Phi(\vec{r})>_{0} \\
=\delta q\left(\log \left|w-w^{\prime}\right|-\log \left|w^{\prime}-w+\left(w^{\prime 2}-1\right)^{1 / 2}-\left(w^{2}-1\right)^{1 / 2}\right|\right. \\
\left.+\log \left|w^{\prime}-w^{*}+\left(w^{\prime 2}-1\right)^{1 / 2}+\left(\left(w^{2}-1\right)^{1 / 2}\right)^{*}\right|-\log \left|w^{\prime}+\left(w^{\prime 2}-1\right)^{1 / 2}\right|\right)
\end{gathered}
$$

where

$$
w=x+i y \quad \text { and } \quad w^{\prime}=x^{\prime}+i y^{\prime}
$$

and we have used the formula

$$
1 /\left(w+\left(w^{2}-1\right)^{1 / 2}\right)=w-\left(w^{2}-1\right)^{1 / 2}
$$

Substituting (2.9) in (2.6), it remains to perform the derivatives. Now, for $-1<x<1$ and $0<y \ll 1$

$$
\left(w^{2}-1\right)^{1 / 2} \sim\left|x^{2}-1\right|^{1 / 2} i e^{i x y /\left(x^{2}-1\right)}
$$

so differentiating (2.9) with respect to $y$ gives

$$
\begin{aligned}
& \left.\frac{\partial}{\partial y}\left(<\Phi(\vec{r})>-<\Phi(\vec{r})>_{0}\right)\right|_{y=0^{+}} \\
& \quad=\delta q\left(-\operatorname{Re} \frac{i}{w^{\prime}-x}-\frac{2}{\left|1-x^{2}\right|^{1 / 2}} \operatorname{Re}\left[\frac{u}{w^{\prime}+\left(w^{\prime 2}-1\right)^{1 / 2}-u}\right]\right)
\end{aligned}
$$

where

$$
u:=x+i\left|x^{2}-1\right|^{1 / 2}
$$

Using (2.11) with $w, x, y$ replaced by $w^{\prime}, x^{\prime}, y^{\prime},(2.12)$ then gives

$$
\begin{aligned}
& \left.\frac{\partial}{\partial y} \frac{\partial}{\partial y^{\prime}}\left(<\Phi(\vec{r})>-<\Phi(\vec{r})>_{0}\right)\right|_{y=0^{+}, y^{\prime}=0^{+}} \\
& \quad=\delta q\left(-\frac{1}{\left(x^{\prime}-x\right)^{2}}+\frac{2}{\left|1-x^{2}\right|^{1 / 2}\left|1-x^{\prime 2}\right|^{1 / 2}} \operatorname{Re}\left[\frac{u^{\prime} u}{\left(u^{\prime}-u\right)^{2}}\right]\right)
\end{aligned}
$$

where

$$
u^{\prime}:=x^{\prime}+i\left|x^{\prime 2}-1\right|^{1 / 2}
$$

Also, since for $-1<x^{\prime}<1$ and $-1 \ll y<0$

$$
\left(w^{\prime 2}-1\right)^{1 / 2} \sim-\left|x^{\prime 2}-1\right|^{1 / 2} i e^{i x^{\prime} y^{\prime} /\left(x^{\prime 2}-1\right)},
$$


we have

$$
\begin{aligned}
& \left.\frac{\partial}{\partial y} \frac{\partial}{\partial y^{\prime}}\left(<\Phi(\vec{r})>-<\Phi(\vec{r})>_{0}\right)\right|_{y=0^{+}, y^{\prime}=0^{-}} \\
& \quad=\delta q\left(-\frac{1}{\left(x^{\prime}-x\right)^{2}}+\frac{2}{\left|1-x^{2}\right|^{1 / 2}\left|1-x^{\prime 2}\right|^{1 / 2}} \operatorname{Re}\left[\frac{u^{\prime *} u}{\left(u^{\prime *}-u\right)^{2}}\right]\right)
\end{aligned}
$$

We now substitute the evaluated derivatives (2.15) and (2.18) in (2.6) to obtain for the charge-charge correlation

$$
\begin{aligned}
& <\sigma(x) \sigma\left(x^{\prime}\right)>^{T} \\
& =\frac{1}{\beta \pi^{2}\left|1-x^{2}\right|^{1 / 2}\left|1-x^{\prime 2}\right|^{1 / 2}} \operatorname{Re}\left[\frac{u^{\prime} u}{\left(u^{\prime}-u\right)^{2}}+\frac{u^{\prime *} u}{\left(u^{\prime *}-u\right)^{2}}\right]
\end{aligned}
$$

Straightforward simplification of the r.h.s. of (2.19) using the definitions (2.14) and (2.16) of $u$ and $u^{\prime}$ reclaims (2.1).

\section{Ensembles of unitary random matrices}

The Dyson circular ensembles of unitary random matrices (see e.g. [1]) have eigenvalue probability density function

$$
\prod_{1 \leq j<k \leq N}\left|e^{i \theta_{k}}-e^{i \theta_{j}}\right|^{\beta}
$$

where $\beta=1,2$ or 4 according to the ensemble being invariant under orthogonal, unitary or symplectic similarity transformations respectively. The probability density is identical, up to a multiplicative constant, to the Boltzmann factor of a one-component log-potential Coulomb system confined to a unit circle. It is particularly simple to apply the argument of Section 2 to this setting and thus deduce that the corresponding two-point correlation $\rho^{T}\left(\theta, \theta^{\prime}\right)$ satisfies the universal formula

$$
\lim _{N \rightarrow \infty} \frac{N}{2 \pi} \int_{\theta}^{\theta^{\prime}+2 \pi / N} d \phi \rho_{N}^{T}(\theta, \phi)=-\frac{1}{\beta(2 \pi)^{2} \sin ^{2}\left(\left(\theta-\theta^{\prime}\right) / 2\right)}
$$

Let us briefly indicate the required working.

First, since the domain is a circle, in polar coordinates the perpendicular component of the electric field is the $r$-component, and thus the analogue of (2.4) is

$$
E_{r}^{+}(\theta)-E_{r}^{-}(\theta)=2 \pi \sigma(\theta)
$$

Let us now assume that the source point $\overrightarrow{r^{\prime}}$ is outside the unit circle. Then, if the field point $\vec{r}$ is also outside the circle, the total potential created is the same as that with the circle replaced 
by a unit conducting disk and is thus given by (2.7). Subtracting the charge-charge portion of this potential then gives

$$
<\Phi(\vec{r})>-<\Phi(\vec{r})>_{0}=\delta q\left(\log \left|z^{\prime}-1 / z^{*}\right|-\log \left|z^{\prime}\right|\right)
$$

For the field point inside the unit circle, there is no net potential due to the source point outside the circle. Subtracting the charge-charge potential gives in this case

$$
<\Phi(\vec{r})>-<\Phi(\vec{r})>_{0}=\delta q \log \left|z^{\prime}-z\right|
$$

The analogue of (2.6) can now easily be evaluated to derive the r.h.s. of (3.2).

The result (3.2) can be used to derive for random unitary matrices (or equivalently, logpotential Coulomb systems on a circle) the analogue of the global fluctuation formula (2.6) . For general systems of point particles on a unit circle

$$
\begin{aligned}
& \lim _{N \rightarrow \infty} \operatorname{var}\left[\sum_{j=1}^{N} f\left(x_{j}\right)\right] \\
& \quad=\lim _{N \rightarrow \infty} \int_{0}^{2 \pi} d \theta \int_{0}^{2 \pi} d \theta^{\prime} f(\theta) f\left(\theta^{\prime}\right)\left(\rho_{N}^{T}\left(\theta-\theta^{\prime}\right)+\rho \delta\left(\sin \left(\theta-\theta^{\prime}\right) / 2\right)\right)
\end{aligned}
$$

where $\rho:=N / 2 \pi$ and the delta denotes the Dirac delta function. Introducing the Fourier series

$$
\lim _{N \rightarrow \infty}\left(\rho_{N}^{T}\left(\theta-\theta^{\prime}\right)+\rho \delta\left(\sin \left(\theta-\theta^{\prime}\right) / 2\right)\right)=\sum_{n=-\infty}^{\infty} s_{n} e^{i\left(\theta-\theta^{\prime}\right) n}
$$

this reads

$$
\lim _{N \rightarrow \infty} \operatorname{var}\left[\sum_{j=1}^{N} f\left(\theta_{j}\right)\right]=\sum_{n=-\infty}^{\infty} s_{n}\left|f_{n}\right|^{2}
$$

where

$$
f_{n}:=\int_{0}^{2 \pi} d \theta f(\theta) e^{i n \theta}
$$

The coefficients $s_{n}$ are given by the usual inversion formula of (3.7). In the inversion formula we replace $\rho^{T}(\theta)$ by its smoothed value (3.2) written as a second derivative:

$$
\rho^{T}(\theta)=\frac{1}{\pi^{2} \beta} \frac{d^{2}}{d \theta^{2}} \log |\sin \theta / 2|, \quad \theta \neq 0
$$

We then calculate the coefficients by a formal differentiation by parts:

$$
\begin{aligned}
s_{n} & =\frac{1}{\pi^{2} \beta} \frac{1}{2 \pi} \int_{-\pi}^{\pi} d \theta\left(\frac{d^{2}}{d \theta^{2}} \log |\sin \theta / 2|\right) e^{i n \theta} \\
& =\frac{1}{\pi^{2} \beta}\left(-n^{2}\right) \frac{1}{2 \pi} \int_{-\pi}^{\pi} d \theta(\log |\sin \theta / 2|) e^{i n \theta} \\
& =\frac{|n|}{2 \pi^{2} \beta}
\end{aligned}
$$


Hence, after substituting (3.11) in (3.8), we obtain the desired global fluctuation formula

$$
\lim _{N \rightarrow \infty} \operatorname{var}\left[\sum_{j=1}^{N} f\left(\theta_{j}\right)\right]=\frac{1}{2 \pi^{2} \beta} \sum_{n=-\infty}^{\infty}|n|\left|f_{n}\right|^{2}
$$

A recent exact calculation [14] allows the formula (3.2) to be illustrated for the one-component log-gas on a unit circle at all even values of $\beta$. The exact calculation gives that the two particle distribution function $\rho_{N}(0, \theta)$ for this system is equal to a $\beta$-dimensional integral [14, eq. (3.15)円 with $\gamma=\beta]$ :

$$
\begin{gathered}
\rho_{N}(0, \theta)=\frac{N(N-1)}{L^{2}}(2 \sin \pi \theta / L)^{\beta} B_{N}(\beta) e^{-\pi i \beta \theta(N-2) / L} \\
\times \int_{[0,1]^{\beta}} d t_{1} \ldots d t_{\beta} \prod_{j=1}^{\beta}\left[1-\left(1-e^{2 \pi i \theta / L}\right) t_{j}\right]^{N-2} D_{2 / \beta, 2 / \beta, 4 / \beta}\left(t_{1}, \ldots, t_{\beta}\right)
\end{gathered}
$$

where

$$
D_{2 / \beta, 2 / \beta, 4 / \beta}\left(t_{1}, \ldots, t_{\beta}\right):=\prod_{j=1}^{\beta}\left[\left(1-t_{j}\right) t_{j}\right]^{1-2 / \beta} \prod_{1 \leq j<k \leq \beta}\left|t_{k}-t_{j}\right|^{4 / \beta}
$$

and $L=2 \pi$. The exact value of the normalization $B_{N}(\beta)$, which is given explicitly in [14], is not required for our discussion below.

We seek to expand (3.13) for large- $N$ so that the integral on the l.h.s of (3.2) can be evaluated. In the large- $N$ limit we see that the $N$-dependent factors of the integrand in (3.13) take on their maximum values at $t_{j}=0$ and $t_{j}=1$. The large- $N$ expansion can be obtained by expanding the integrand about the maximum values. Note in particular that for $t_{j} \sim 0$ and $L=2 \pi$

$$
\left[1-\left(1-e^{2 \pi i \theta / L}\right) t_{j}\right]^{N-2} \sim e^{-(N-2)\left(1-e^{i \theta}\right) t_{j}}
$$

while for $t_{j} \sim 1$ and $L=2 \pi$

$$
\left[1-\left(1-e^{2 \pi i \theta / L}\right) t_{j}\right]^{N-2} \sim e^{i(N-2) \theta} e^{-(N-2)\left(1-e^{-i \theta}\right)\left(1-t_{j}\right)}
$$

At this stage we observe that the problem is very similar to one we have solved recently [12]. The solved problem is the task of calculating the large- $x$ asymptotic expansion of the thermodynamic limit form of the two-particle distribution. Now in the thermodynamic limit $N, L \rightarrow \infty(N / L=\eta$ fixed) (3.13) becomes

$$
\begin{gathered}
\rho(0, x)=\eta^{2}(\eta x)^{\beta} A(\beta) e^{-\pi i \beta \eta x} \int_{[0,1]^{\beta}} d t_{1} \ldots d t_{\beta} \prod_{j=1}^{\beta} e^{2 \pi i t_{j} \eta x} \\
\times D_{2 / \beta, 2 / \beta, 4 / \beta}\left(t_{1}, \ldots, t_{\beta}\right)
\end{gathered}
$$

\footnotetext{
${ }^{1}$ This equation is missing a factor of $e^{-\pi i \eta \gamma(N-2) / N}$; c.f. eq. (3.2) of [14].
} 
from which the large- $x$ asymptotic expansion can be shown to be of the form

$$
\begin{gathered}
\rho^{T}(0, x) \sim \eta^{2}\left[-\frac{1}{\beta(\pi \eta x)^{2}}+\frac{a^{(4)}}{(\eta x)^{4}}+\ldots+\sum_{n=1}^{\beta / 2} \frac{\cos 2 \pi \eta x n}{(\eta x)^{4 n^{2} / \beta}}\left(b_{n}^{(0)}+\frac{b_{n}^{(2)}}{(\eta x)^{2}}+\ldots\right)\right. \\
\left.\quad+\sum_{n=1}^{\beta / 2} \frac{\sin 2 \pi \eta x n}{(\eta x)^{4 n^{2} / \beta+1}}\left(c_{n}^{(0)}+\frac{c_{n}^{(2)}}{(\eta x)^{2}}+\ldots\right)\right]
\end{gathered}
$$

The method used in [12] to compute (3.18) was to expand the integrand in (3.17) about the endpoints $t_{j}=0,1$. We thus see that this problem is almost identical to the task of computing the large- $N$ expansion of (3.13).

Indeed, by comparing (3.13) and (3.17) and examining (3.15) and (3.16) we see that the large- $N$ expansion of (3.13) is identical to the large- $x$ expansion of (3.17) and is thus given by (3.18) provided we make the replacements

$$
\pi \eta x \mapsto 2 N \sin \theta / 2, \quad \eta \mapsto N / 2 \pi
$$

Substituting the resulting expansion in the l.h.s. of (3.2) we see that the oscillatory terms all integrate to zero, and all but the first of the non-oscillatory terms vanish in the $N \rightarrow \infty$ limit. The first non-oscillatory term gives the r.h.s. of (3.2) as required.

\section{Acknowledgement}

I thank B. Jancovici for some useful remarks. 


\section{References}

[1] M.L. Mehta, Random Matrices, 2nd ed. (Academic Press, San Diego, 1991).

[2] E. Brézin and A. Zee, Nucl. Phys. B 402 (1993) 613.

[3] B. Eynard, hep-th/9401165, to appear Nucl. Phys. B.

[4] C.W.J. Beenakker, preprint cond-mat/9310010, to appear Nucl. Phys. B.

[5] B.V. Bronk, J. Math. Phys. 6 (1965) 228.

[6] T. Nagao and M. Wadati, J. Phys. Soc. Japan 61 (1992) 78, 1911; T. Nagao and P.J. Forrester, preprint.

[7] P.J. Forrester, Nucl. Phys. B 402 (1993) 709.

[8] C.A. Tracy and H. Widom, Commun. Math. Phys. 161 (1994) 289.

[9] C.W.J. Beenakker, Phys. Rev. Lett. 70 (1993) 1155.

[10] A.M.S. Macêde and J.T. Chalker, Phys. Rev. B 49 (1994) 4695.

[11] B. Jancovici and P.J. Forrester, to appear Phys. Rev. B.

[12] P.J. Forrester, Phys. Lett. A 179 (1993) 127.

[13] W.K.H. Panofsky and M. Phillips, Classical electricity and magnetism, 2nd ed. (AddisonWesley, 1972).

[14] P.J. Forrester, Nucl. Phys. B 416 (1994) 377. 